\title{
Progress in Gauge-Higgs Unification on the Lattice
}

\author{
Francesco Knechtli*, Kyoko Yoneyama ${ }^{\dagger}$, Peter Dziennik \\ Department of Physics, Bergische Universität Wuppertal \\ Gaussstr. 20, D-42119 Wuppertal, Germany \\ E-mail: knechtli@physik.uni-wuppertal.de, \\ yoneyama@physik.uni-wuppertal.de, dziennikeuni-wuppertal.de
}

\section{Nikos Irges}

Department of Physics, National Technical University of Athens

Zografou Campus, GR-15780 Athens, Greece

E-mail: irges@mail.ntua.gr

\begin{abstract}
We study a five-dimensional pure $S U(2)$ gauge theory formulated on the orbifold and discretized on the lattice by means of Monte Carlo simulations. The gauge symmetry is explicitly broken to $U(1)$ at the orbifold boundaries. The action is the Wilson plaquette action with a modified weight for the boundary $U(1)$ plaquettes. We study the phase transition and present results for the spectrum and the shape of the static potential on the boundary. The latter is sensitive to the presence of a massive Z-boson, in good agreement with the directly measured Z-boson mass. The results may support an alternative view of the lattice orbifold (stemming from its mean-field study) as a $5 \mathrm{~d}$ bosonic superconductor.
\end{abstract}

31st International Symposium on Lattice Field Theory - LATTICE 2013

July 29 - August 3, 2013

Mainz, Germany

\footnotetext{
${ }^{*}$ Speaker. FK thanks CERN for hospitality.

† Speaker.
} 


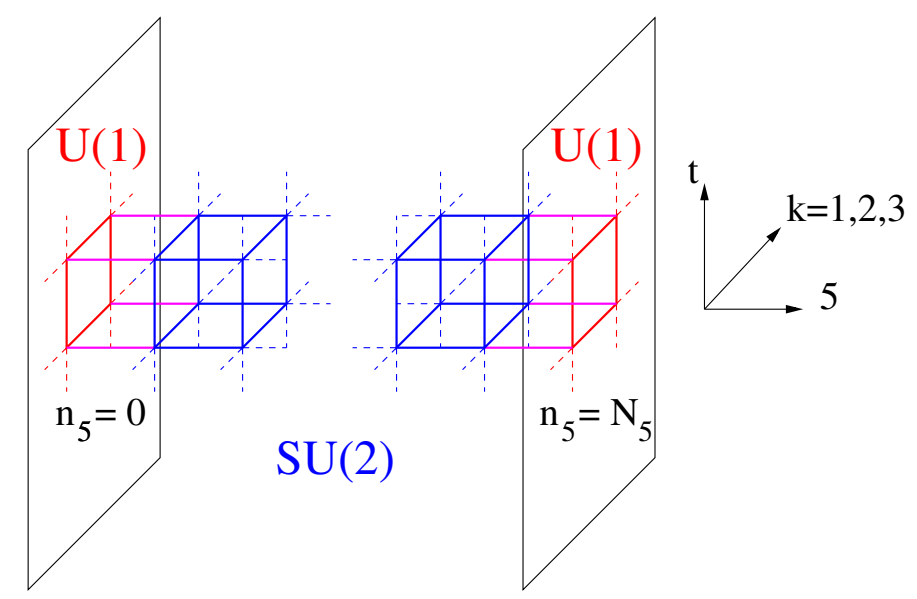

Figure 1: Schematic representation of the 5 d lattice where the $S U(2)$ orbifolded gauge theory is defined.

\section{The $S U(2)$ lattice orbifold and its symmetries}

We use a five-dimensional (5d) anisotropic Euclidean lattice with $T \times L^{3} \times\left(N_{5}+1\right)$ points. The lattice spacing is $a_{4}$ in the four-dimensional (4d) hyperplanes orthogonal to the extra dimension and $a_{5}$ along the extra dimension. The gauge links $U_{M}(n) \in S U(2)$ connect the lattice points $n+\hat{M}$ with $n$. The Euclidean index $M$ runs over the temporal $M=0$ and spatial $M=k, 5(k=1,2,3)$ directions. We will also use Greek indices to denote the $\mu=0, k$ directions. The lattice is assumed to be periodic except for the extra dimension, which is an interval with boundaries originating from an orbifold projection [1]. The physical length of the extra dimension is $\pi R=N_{5} a_{5}$. We employ the anisotropic Wilson plaquette action which is defined as

$$
S_{W}^{\mathrm{orb} .}=\frac{\beta}{2}\left[\frac{1}{\gamma} \sum_{4 \mathrm{~d} \mathrm{p}} w \operatorname{Retr}\left\{I-P_{\mu v}(n)\right\}+\gamma \sum_{5 \mathrm{dp}} \operatorname{Retr}\left\{I-P_{\mu 5}(n)\right\}\right],
$$

where the weight factor is $w=1 / 2$ for the boundary plaquettes and $w=1$ otherwise. Only counterclockwise oriented plaquettes $P_{M N}$ are summed over and $I$ is the identity matrix. The anisotropy parameter $\gamma$ is in the classical continuum limit $\gamma=a_{4} / a_{5}$. Instead of $(\beta, \gamma)$ we will use the equivalent parameter pair $\left(\beta_{4}=\beta / \gamma, \beta_{5}=\beta \gamma\right)$. Along the extra dimension with coordinate $n_{5} \in\left[0, N_{5}\right]$ the orbifold projection specifies Dirichlet boundary conditions

$$
U_{\mu}(n)=g U_{\mu}(n) g^{-1} \Rightarrow U_{\mu}(n)=\mathrm{e}^{\phi(n) g} \in U(1)
$$

at $n_{5}=0$ and $n_{5}=N_{5}$ with the $S U(2)$ projection matrix

$$
g=-i \sigma^{3}
$$

Thus, at the interval ends, the gauge symmetry is explicitly broken to a $U(1)$ subgroup of $S U(2)$ which is left invariant by group conjugation with $g$. The lattice of the $S U(2)$ orbifolded gauge theory is schematically represented in Fig. 1. There are three types of gauge links: $4 \mathrm{~d} U(1)$ links contained in the two boundaries at $n_{5}=0$ and $n_{5}=N_{5}$, hybrid extra dimensional links (with one end 
on a boundary transforming under $U(1)$ and the other end in the bulk transforming under $S U(2)$ ) and the remaining bulk $S U(2)$ links.

In addition to the local gauge symmetry, the orbifold theory possesses the following global symmetries

$$
Z \otimes F \otimes \mathscr{F} .
$$

$Z$ is a center transformation in the 4 d hyperplanes by an element of the center $\mathbb{Z}_{2}=\{ \pm I\}$ of $S U(2)$. $F$ is a reflection with respect to the middle of the orbifold interval $n_{5}=N_{5} / 2$. The fixed point symmetry is $\mathscr{F}=\mathscr{F}_{L} \oplus \mathscr{F}_{R}$. On the $L$ ("left") boundary at $n_{5}=0, \mathscr{F}_{L}$ is defined as

$$
\begin{aligned}
& U_{5}\left(n_{5}=0\right) \rightarrow g_{F}^{-1} U_{5}\left(n_{5}=0\right) \\
& U_{v}\left(n_{5}=0\right) \rightarrow g_{F}^{-1} U_{v}\left(n_{5}=0\right) g_{F}
\end{aligned}
$$

and on the $R$ ("right") boundary at $n_{5}=N_{5}, \mathscr{F}_{R}$ is defined as

$$
\begin{aligned}
U_{5}\left(n_{5}=N_{5}-1\right) & \rightarrow U_{5}\left(n_{5}=N_{5}-1\right) g_{F} \\
U_{v}\left(n_{5}=N_{5}\right) & \rightarrow g_{F}^{-1} U_{v}\left(n_{5}=N_{5}\right) g_{F} .
\end{aligned}
$$

In order that $\mathscr{F}_{L(R)}$ are consistent symmetry transformations, a boundary $U(1)$ gauge link should remain in the $U(1)$ group after conjugation by $g_{F}$. Moreover, the transformations have to commute with the orbifold projection and $g_{F}$ has to satisfy [3]

$$
g g_{F}=g_{F} g z_{G},
$$

where $z_{G}$ is an element of the center of $S U(2)$, i.e. $z_{G}= \pm I$. If $z_{G}=-I$ or equivalently

$$
\left\{g, g_{F}\right\}=0,
$$

for example $g_{F}=\mathrm{e}^{i \theta}\left(-i \sigma^{2}\right)$, the fixed point transformations $\mathscr{F}_{L(R)}$ are the stick symmetries introduced in [3] and we denote them by $\mathscr{S}_{L(R)}$. If $z_{G}=I$ or equivalently $\left[g, g_{F}\right]=0$, then the transformations $\mathscr{F}_{L(R)}$ are global gauge transfomations.

In order to build lattice operators for the scalar (Higgs) and vector (gauge boson) particles, see [2], we will use the boundary-to-boundary-line

$$
l=\prod_{n_{5}=0}^{N_{5}-1} U_{5}\left(n_{5}\right) .
$$

and the orbifolded Polyakov loop ${ }^{1}$

$$
P_{L}=\lg l^{\dagger} g^{-1}
$$

Higgs operators, with spin $J=0$, charge conjugation $C=1$ and spatial parity $P=1$, are defined by

$$
\operatorname{tr} P_{L}, \quad \operatorname{tr} \Phi_{L} \Phi_{L}^{\dagger}
$$

where $\Phi_{L}=1 /\left(4 N_{5}\right)\left[P_{L}-P_{L}^{\dagger}, g\right]$. Inspired by [4], we define a gauge boson operator, with $J=1$, $C=-1$ and $P=-1$, by

$$
\operatorname{tr} Z_{L k}, \quad Z_{L k}(n)=g U_{k}(n) \alpha_{L}(n+\hat{k}) U_{k}^{\dagger}(n), \alpha_{L}(n),
$$

\footnotetext{
${ }^{1}$ The subscript $L(R)$ indicates that an operator is defined on the $L(R)$ boundary.
} 


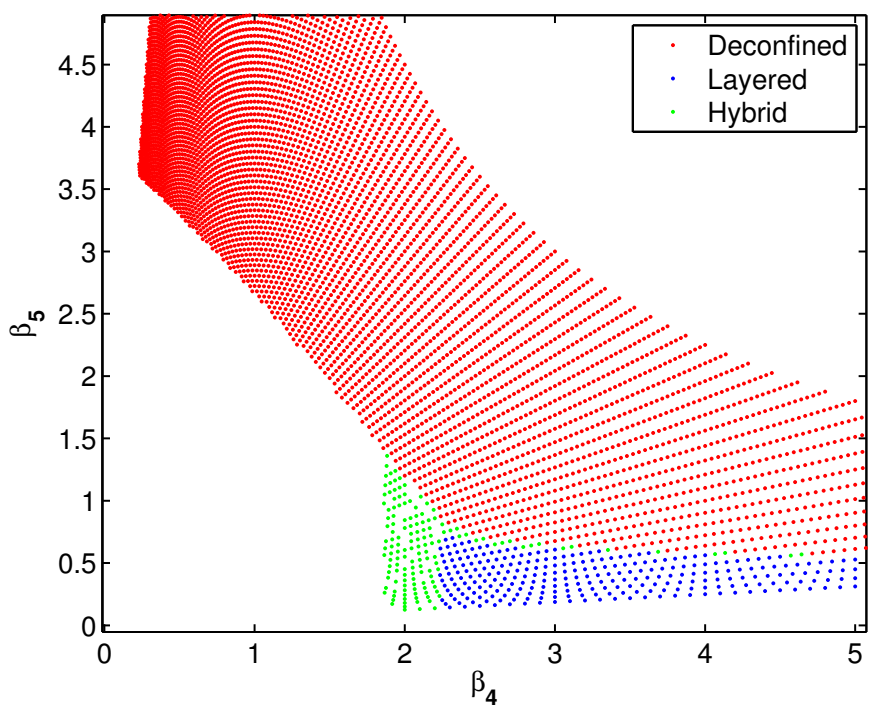

Figure 2: The mean-field phase diagram. The size of the extra dimension is $N_{5}=12$. Each point represents the solution of a numerical iterative process.

where $\alpha_{L}=\Phi_{L} / \sqrt{\operatorname{det} \Phi_{L}}$. Note that while $\operatorname{tr} P_{L}$ is odd under both stick symmetries $\mathscr{S}_{L}$ and $\mathscr{S}_{R}$, $\operatorname{tr} Z_{L}$ is odd under $\mathscr{S}_{L}$ and even under $\mathscr{S}_{R}$.

Another quantity which will be used in particular to study dimensional reduction from five to four dimensions is the static potential $V(r)$ extracted from Wilson loops. We take the latter to be defined in a $4 \mathrm{~d}$ hyperplane, so the static potential depends on the coordinate $n_{5}$.

In order to build a larger variational basis, the links used to construct the Higgs and gauge boson operators and the Wilson loops are smeared with a HYP smearing [5] which does not use the temporal links and is adapted for the orbifold. In particular the smearing parameters are set to $\alpha_{1}=0.5, \alpha_{2}=0.4$ and $\alpha_{3}=0.2$ and the spatial links in the $4 \mathrm{~d}$ hyperplanes are not smeared along the extra dimension.

\section{Phase diagram}

\subsection{Mean-field}

Fig. 2 shows the phase diagram based on the solutions obtained for the mean-field background $\bar{v}_{0}$. Due to the orbifold boundaries, we distinguish a background $\bar{v}_{0}\left(n_{5}\right), n_{5}=0,1, \ldots, N_{5}$ in the $4 \mathrm{~d}$ hyperplanes and $\bar{v}_{0}\left(n_{5}+1 / 2\right), n_{5}=0,1, \ldots, N_{5}-1$ along the extra dimension. For the details of the mean-field formulation we refer to [6]. The mean-field phase diagram in Fig. 2 has four phases:

1. $\bar{v}_{0}\left(n_{5}\right)=0, \bar{v}_{0}\left(n_{5}+1 / 2\right)=0$ : confined phase (white color);

2. $\bar{v}_{0}\left(n_{5}\right) \neq 0, \bar{v}_{0}\left(n_{5}+1 / 2\right) \neq 0$ : deconfined phase (red color);

3. $\bar{v}_{0}\left(n_{5}\right) \neq 0, \bar{v}_{0}\left(n_{5}+1 / 2\right)=0$ : layered phase, cf. [7] (blue color);

4. $\bar{v}_{0}\left(n_{5}=0\right) \neq 0, \bar{v}_{0}\left(n_{5}=N_{5} / 2\right)=0$ : hybrid phase (green color). 


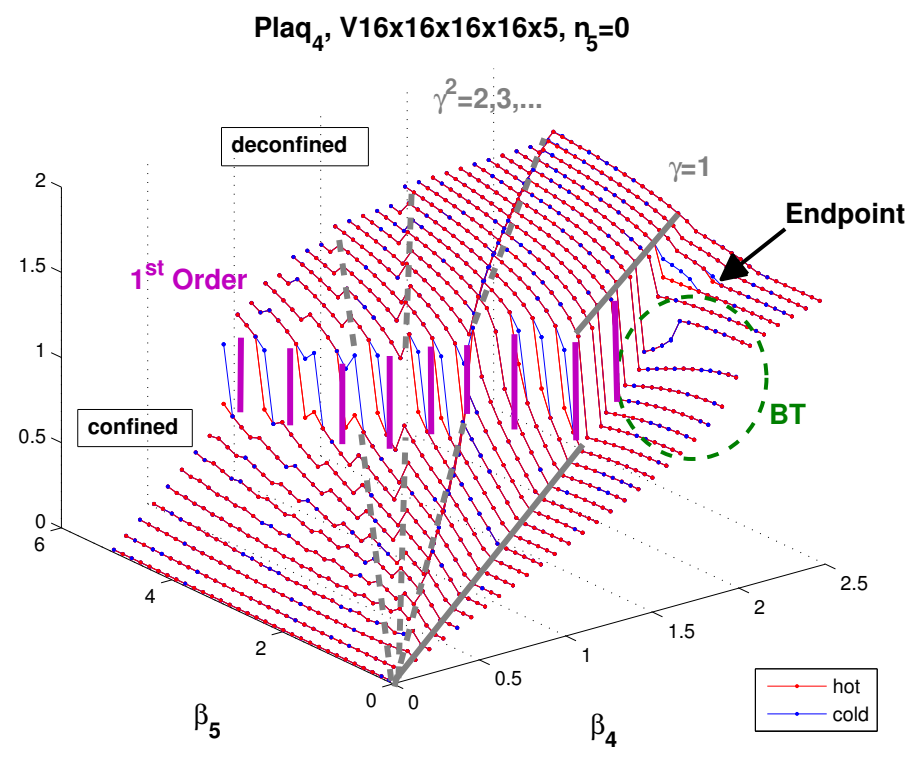

Figure 3: Overview of the Monte Carlo phase diagram of the $4 \mathrm{~d}$ boundary plaquette on the $16^{4} \times 5$ orbifold. Hot (red) and cold (blue) starts.

The mean-field sees only bulk phase transitions and is not sensitive to compactification effects. Approaching the phase boundary from the deconfined phase, we define the critical exponent $v$ of the inverse correlation length, which is given by the Higgs mass $M_{H}=a_{4} m_{H}(\beta)=\left(1-\beta_{c} / \beta\right)^{v}$ (and is measured from the Euclidean time correlator of the operator in Eq. (1.13)). For $\gamma>0.6$, we measure $v=1 / 4$, the Higgs mass $M_{H}$ and the background do not vanish as the phase transition is approached. This means that the phase transition is of first order (the lattice spacing does not go to zero). For $\gamma \lesssim 0.6$, the layered phase appears at the phase boundary and $v$ becomes $1 / 2$. Moreover the Higgs mass and the background tend to zero approaching the boundary of the deconfined/layered phases. This is consistent with a second order phase transition. Indeed in [6], lines of constant physics (LCPs) have been constructed along which the continuum limit was taken. In particular on a LCP with $\rho_{H Z}=m_{H} / m_{Z}=1.38$ (which is the current experimental value) we find that the Higgs mass is finite without supersymmetry and predict a $Z^{\prime}$ state of mass $m_{Z^{\prime}} \simeq 1 \mathrm{TeV}$. The mean-field calculations show that a LCP with $\rho_{H Z}=1.38$ at $\gamma \geq 1$ does not exist.

\subsection{Monte Carlo}

Fig. 3 shows the phase diagram of the $4 \mathrm{~d}$ plaquette on the boundary $n_{5}=0$ in the $\left(\beta_{4}, \beta_{5}\right)$ plane. We simulated a $16^{4} \times 5$ orbifold in the parameter region $\beta_{4} \in[0.3,2.5]$ and $\beta_{5} \in[0.3,5]$. For each parameter point we did two runs (hot and cold start) with 150 thermalization and 400 measurement steps. Two steps are separated by one heatbath and 8 overrelaxation update sweeps.

There is a bulk phase transition of first order ${ }^{2}$. It is signalled by a hysteresis and is marked by the magenta vertical lines in Fig. 3. At $\gamma=1$ the hysteresis is between $\beta=1.55$ and $\beta=1.65$, it is wider than on the torus, cf. [9].

\footnotetext{
${ }^{2}$ The corresponding transition with periodic boundary conditions along the extra dimension (torus) was studied in [9], see also [10].
} 


\begin{tabular}{llll}
\hline & $\beta_{4}$ & $\beta_{5}$ & Peak Height \\
\hline $\mathrm{L}=12$ & $2.248 \pm 0.003$ & $1.41 \pm 0.01$ & $1.17 \pm 0.15$ \\
$\mathrm{~L}=16$ & $2.3575 \pm 0.0005$ & $1.41 \pm 0.01$ & $0.8 \pm 0.1$ \\
\hline
\end{tabular}

Table 1: The "end-point" of the bulk first order phase transition for lattices of size $T=L=12, T=L=16$ and $N_{5}=4$. The last column shows the peak of the susceptibility of the $4 \mathrm{~d}$ plaquette at $n_{5}=2$.

\begin{tabular}{ll}
\multicolumn{2}{l}{$\beta_{5}=1.24:$} \\
\hline $\mathrm{V}$ & $\beta_{4}$ \\
\hline $8^{4} \times 5$ & $2.0592 \pm 0.0016$ \\
$12^{4} \times 5$ & $2.0697 \pm 0.0007$ \\
$16^{4} \times 5^{*}$ & $2.0740 \pm 0.0010$ \\
\hline
\end{tabular}

Table 2: The BT $\beta_{4}$ values at $\beta_{5}=1.24$. The error is determined by the width of the susceptibility peak. The $L=16^{*}$ lattice shows a clear hysteresis and its error is therefore taken to be half of the hysteresis width.

In general there are jumps of the plaquette along lines where $\gamma^{2} \in \mathbb{N}^{+}$, which are marked by the gray lines in Fig. 3. Corresponding to these jumps of the plaquette value, we also find jumps of the plaquette susceptibility but no peaks of the latter. The presence of these jumps does not depend on the lattice size.

The $\gamma<1$ region is interesting because the mean-field analysis predicts that the bulk phase transition changes from first to second order, see Section 2.1. Although a second order phase transition has not been found so far in Monte Carlo simulations on the torus, hints for dimensional reduction and for properties resembling those of a layered phase as seen by the mean-field are reported in [9]. Here we find on the orbifold two potential candidates for a second order phase transition, see Fig. 3. One is the "end-point" of the first order bulk phase transition line, where the hysteresis disappears. The other is a new type of transition signalled by an inflection point in the boundary $4 \mathrm{~d}$ plaquette at fixed $\beta_{5}$ as a function of $\beta_{4}$. We call it boundary transition (BT), because it is not visible in the $4 \mathrm{~d}$ plaquette in the middle of the orbifold at $n_{5}=2$. The BT is therefore reminiscent of the hybrid phase found in the mean-field phase diagram of Fig. 2.

Table 1 shows that the position of the "end-point" strongly changes with the $4 \mathrm{~d}$ lattice volume and the peak of the plaquette susceptibility is approximately constant. So we discard it as a lattice artifact.

Instead the position of the BT does not depend much on the volume, see Table 2. We find that the peak of the susceptibility of the boundary plaquette at the BT point increases by a factor of more than two between the volumes $8^{4}$ and $12^{4}$ (the ratio of the volumes is approximately 5). This could be consistent with a second order phase transition. However for the volume $16^{4}$ we find a clear hysteresis which means that the BT transition at $\beta_{5}=1.24$ is of first order.

We mention that the $4 \mathrm{~d}$ plaquette measured in the bulk gives a consistent picture, except that it does not see the BT. Also the phase diagram on a $24^{4} \times 5$ lattice gives the same qualitative picture.

Fig. 4 shows the phase diagram of the absolute value of $\operatorname{tr} P_{L}$ defined in Eq. (1.12). It is obtained 

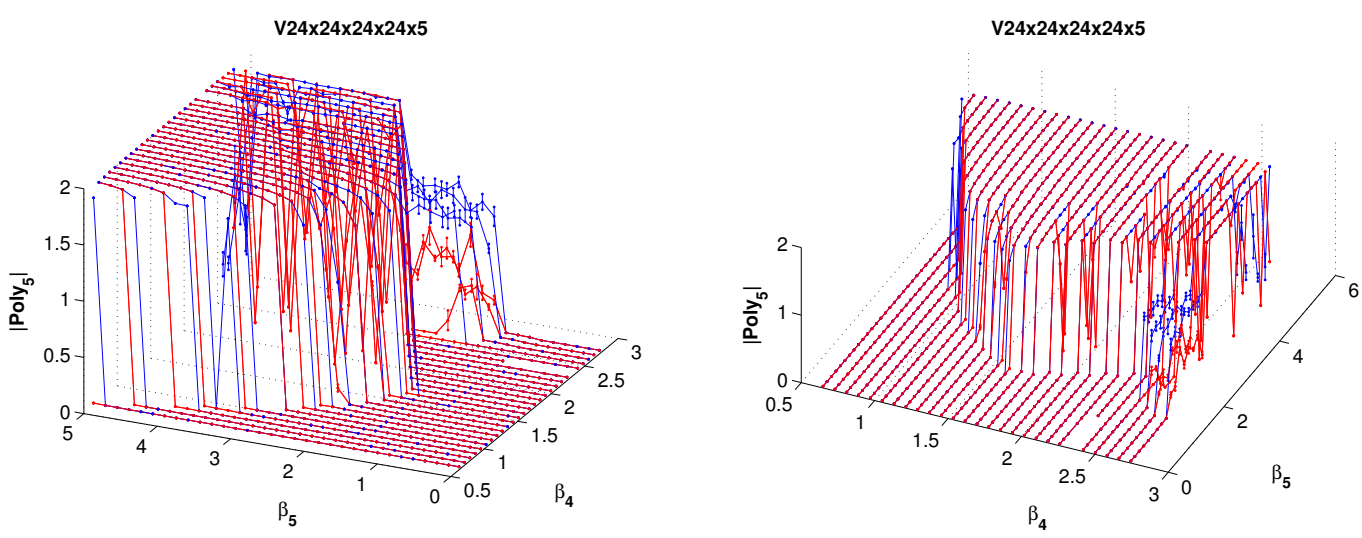

Figure 4: Overview of the Monte Carlo phase diagram of $\left|\mathrm{Poly}_{5}\right|=\left|\operatorname{tr} P_{L}\right|$ on the $24^{4} \times 5$ orbifold. Hot (red) and cold (blue) starts. The two plots show the same data but from different views.

from hot and cold starts each having 250 thermalization and 2000 measurement steps on a $24^{4} \times 5$ orbifold. $\left|\operatorname{tr} P_{L}\right|$ clearly behaves like an order parameter for confinement, separating the confined from the deconfined phases. It vanishes in the confined phase and saturates quickly at the value 2 in the deconfined phase. There is also an intermediate "plateau" for $\beta_{4} \gtrsim 2.5$ for $\beta_{5}$ values larger than at the "end-point" transition (we have indications that for this lattice size the "end-point" moves to such high values $\beta_{4} \approx 2.5$ ). Without taking the absolute value, the sign of $\operatorname{tr} P_{L}$ changes frequently. This is an indication that the two stick symmetries $\mathscr{S}_{L}$ and $\mathscr{S}_{R}$ are both spontaneously broken in the deconfined phase. Unfortunately the observables $\operatorname{tr} Z_{L k}^{2}$, see Eq. (1.14), is too noisy and we cannot display its phase diagram.

\section{Spectrum}

On an isotropic $(\gamma=1)$ orbifolded $64 \times 32^{3} \times 5$ lattice we measure the spectrum close to the bulk phase transition ( $\beta=1.66,1.68)$ using the operators defined in Eq. (1.13) and Eq. (1.14). The statistics is of 2000 measurements separated by two update steps (each update step consists of one heatbath and 16 overrelaxation sweeps). The variational basis is constructed using smeared links with 5, 15 and 30 HYP smearing iterations.

The masses of the Higgs and gauge boson in units of $1 / R$ are shown in Fig. 5. Together with the ground states, also the first excited states were resolved. We find a nonzero gauge-boson mass $m_{Z} \neq 0$ and the value of $m_{Z}$ does not decrease with the lattice size $L$. This implies that the Higgs mechanism is at work. The mass hierarchy is not the one measured by the experiments at CERN, we find $m_{Z}>m_{H}$. The masses of the excited states of the Higgs and the Z-boson are approximately equal.

At $\beta=1.66$ we also measured the static potential on the boundary. The data (blue points) are shown on the left plot of Fig. 6. In order to get a qualitative understanding of the shape of the potential, we perform $4 \mathrm{~d}$ Yukawa (red dashed line), 4d Coulomb (black continued line) and $5 \mathrm{~d}$ Coulomb (green dash-dotted line) global fits ${ }^{3}$. In the Yukawa non-linear fit, the Yukawa mass is a

\footnotetext{
${ }^{3}$ The best $5 \mathrm{~d}$ Yukawa fit has a zero Yukawa mass and is therefore not considered.
} 

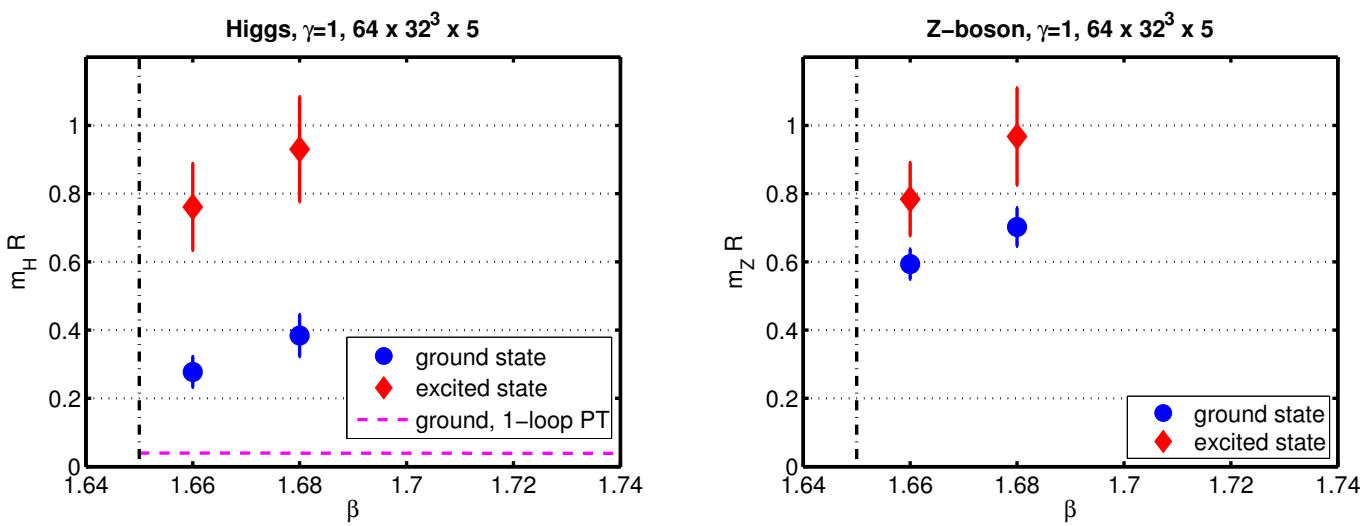

Figure 5: The spectrum for $\gamma=1, L=32$ and $N_{5}=4$ close to the bulk phase transition marked by the vertical dash-dotted line. The masses of the Higgs (gauge boson) ground and excited states are shown on the left (right) plot in units of $1 / R$. The Higgs mass is much higher than its value from the one-loop (continuum) formula $[11,12]$.
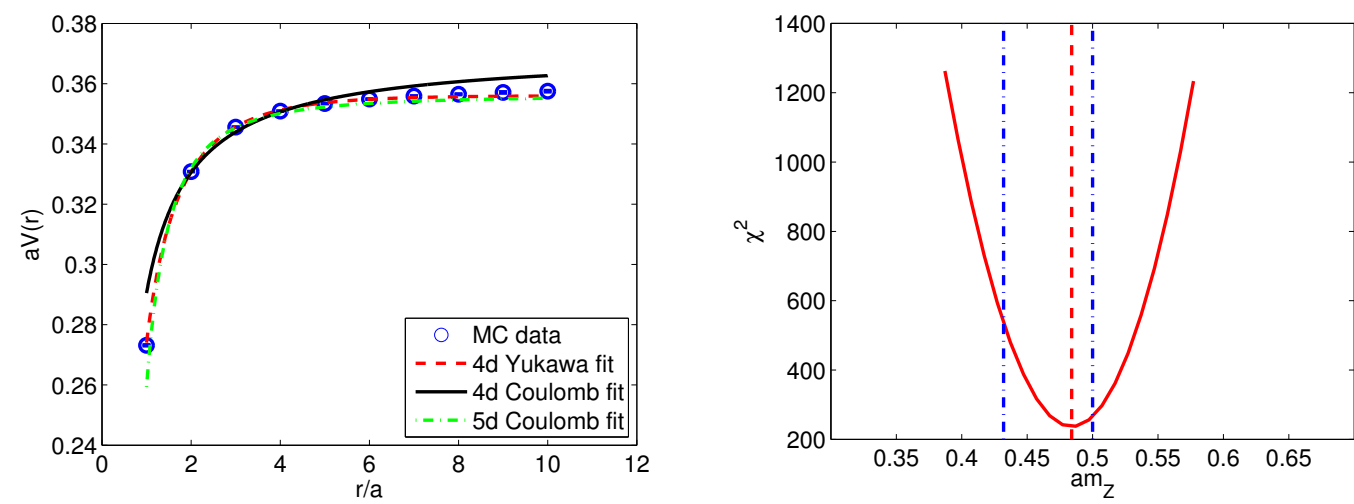

Figure 6: The boundary static potential at $\gamma=1, L=32, N_{5}=4$ and $\beta=1.66$ (left plot) favors the $4 \mathrm{~d}$ Yukawa global fit. The Yukawa mass which minimizes the $\chi^{2}$ (right plot, red vertical dashed line) agrees well with the directly measured value marked by the vertical blue dashed-dotted lines.

fit parameter. The preferred global fit is the $4 \mathrm{~d}$ Yukawa. The $\chi^{2}$ of the $4 \mathrm{~d}$ Yukawa fit is shown on the right plot of Fig. 6 as function of the Yukawa mass $a m_{Z}$. The value of $a m_{Z}$ which minimizes the $\chi^{2}$ is consistent with the directly measured value of $a m_{Z}$, cf. Fig. 5.

After we proved the existence of the Higgs mechanism, by finding a nonzero mass for the $U(1)$ gauge boson, the question of its origin arises. In particular Elitzur's theorem [13] tells that only global symmetries can be spontaneously broken on the lattice and phase transitions are characterized by gauge invariant order parameters. In Section 1 we have identified global symmetries, the fixed point symmetries $\mathscr{F}_{L}$ and $\mathscr{F}_{R}$, which contain the stick symmetries $\mathscr{S}_{L}$ and $\mathscr{S}_{R}$. In the deconfined phase, where we measure the masses, the stick symmetries are spontaneously broken, see Section 2.2. This breaking induces the breaking of the other global symmetries, which are global gauge transformations, this is the origin of the Higgs mechanism. The Polyakov loop $\operatorname{tr} P_{L}$ in Eq. (1.13) is the order parameter for confinement/deconfinement. The deconfinement phase can be a Coulomb or a Higgs phase. We conjecture that the operator $\operatorname{tr} Z_{L k}$ in Eq. (1.14), is the order 


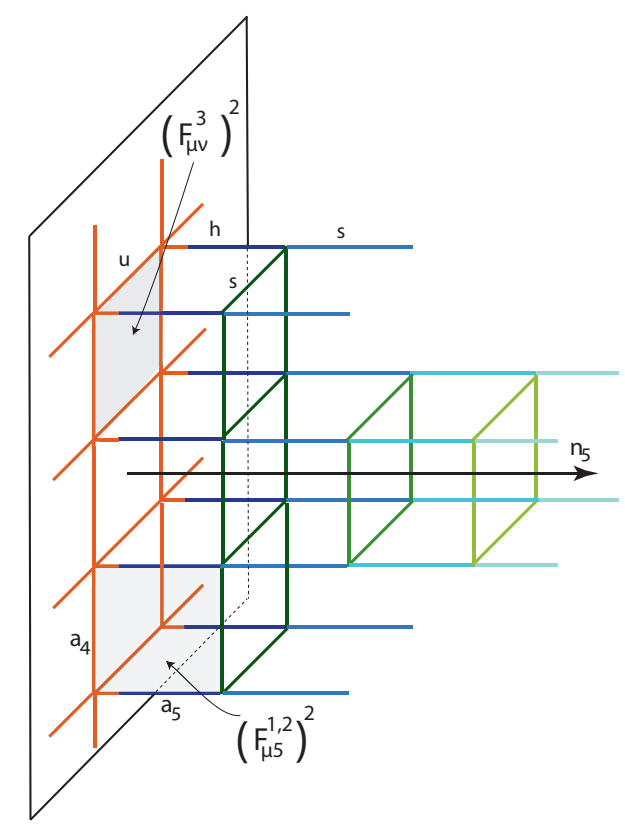

Figure 7: The mean-field background defines a "crystal" in coordinate and gauge space. Links of different colors correspond to different values of the background.

parameter of the Higgs phase, namely the Higgs mechanism happens when $\left\langle\operatorname{tr} Z_{L k}^{2}\right\rangle \neq 0$.

The Higgs mechanism on the orbifold seems to have a different origin than the Hosotani mechanism [14], which was formulated in perturbation theory and works only when fermions are included, see also the lattice study of [15]. The orbifold mechanism of spontaneous symmetry breaking could be related to a bosonic superconductor. The mean-field background breaks translation invariance, like a crystal, see Fig. 7. Gauge fluctuations around the mean-field background are like phonons, the Polyakov loop (Higgs) with $U(1)$ charge 2 is like a Cooper pair and the Higgs mechanism, due to gauge-Higgs interaction, happens like in a superconductor slab, where the photon becomes massive.

\section{Conclusions}

We have presented a Monte Carlo study of the $5 \mathrm{~d} S U(2)$ orbifold, extending previous results from [2]. A second order phase transition is not found. Instead there is a line of bulk first order phase transitions and a new transition for anisotropy $\gamma<1$ which is signalled by the boundary (but not by the bulk) $4 \mathrm{~d}$ plaquette. The boundary transition is also of first order where we did simulations.

The spectrum is measured so far only at $\gamma=1$ for an orbifold with $N_{5}=4$, where we find a massive gauge boson which is heavier than the Higgs scalar. The mass of the gauge boson is consistent with the mass extracted from a $4 \mathrm{~d}$ Yukawa fit to the boundary static potential, thus supporting dimensional reduction. It remains to be seen how the mass hierarchy is at $\gamma<1$, where in the mean-field calculation it was possible to reproduce the experimentally measured masses for the Higgs and gauge boson. 


\section{References}

[1] N. Irges and F. Knechtli, Non-perturbative definition of five-dimensional gauge theories on the $\mathbb{R}^{4} \times S^{1} / \mathbb{Z}_{2}$ orbifold, Nucl. Phys. B 719 (2005) 121 .

[2] N. Irges and F. Knechtli, Lattice gauge theory approach to spontaneous symmetry breaking from an extra dimension, Nucl. Phys. B 775 (2007) 283.

[3] K. Ishiyama, M. Murata, H. So and K. Takenaga, Symmetry and $Z_{2}$ Orbifolding Approach in Five-dimensional Lattice Gauge Theory, Prog. Theor. Phys. 123 (2010) 257.

[4] I. Montvay, Correlations in the SU(2) Fundamental Higgs Model, Phys. Lett. B 150 (1985) 441.

[5] A. Hasenfratz, F. Knechtli, Flavor symmetry and the static potential with hypercubic blocking, Phys. Rev. D 64 (2001) 034504.

[6] N. Irges, F. Knechtli and K. Yoneyama, Mean-Field Gauge Interactions in Five Dimensions II. The Orbifold, Nucl. Phys. B 865 (2012) 541.

[7] Y. K. Fu and H. B. Nielsen, A Layer Phase in a Nonisotropic U(1) Lattice Gauge Theory: Dimensional Reduction a New Way, Nucl. Phys. B 236 (1984) 167.

[8] N. Irges, F. Knechtli and K. Yoneyama, Higgs mechanism near the 5d bulk phase transition, Phys. Lett. B 722 (2013) 378.

[9] F. Knechtli, M. Luz and A. Rago, On the phase structure of five-dimensional SU(2) gauge theories with anisotropic couplings, Nucl. Phys. B 856 (2012) 74.

[10] L. Del Debbio, R. D. Kenway, E. Lambrou and E. Rinaldi, The transition to a layered phase in the anisotropic five-dimensional SU(2) Yang-Mills theory, Phys. Lett. B 724 (2013) 1-3, 133.

[11] G. von Gersdorff, N. Irges and M. Quiros, Bulk and brane radiative effects in gauge theories on orbifolds, Nucl. Phys. B 635 (2002) 127.

[12] H. -C. Cheng, K. T. Matchev and M. Schmaltz, Radiative corrections to Kaluza-Klein masses, Phys. Rev. D 66 (2002) 036005.

[13] S. Elitzur, Impossibility of Spontaneously Breaking Local Symmetries, Phys. Rev. D 12 (1975) 3978.

[14] Y. Hosotani, Dynamical Gauge Symmetry Breaking as the Casimir Effect, Phys. Lett. B 129 (1983) 193.

[15] G. Cossu, H. Hatanaka, Y. Hosotani and J. -I. Noaki, Polyakov loops and the Hosotani mechanism on the lattice, arXiv:1309.4198 [hep-lat ]. 